\title{
Corrosión en soldaduras de aparatos de ortodoncia $^{(\bullet)}$
}

\author{
S.M. Vázquez ${ }^{(*)}$, O. Riesgo ${ }^{(* *)}$ y G.S. Duffó ${ }^{(* * *)}$ \\ Resumen En los últimos años se ha desarrollado considerablemente el estudio de los problemas relacionados \\ con la corrosión de aleaciones dentales a efectos de evitar la utilización de materiales con una \\ deficiente resistencia a la corrosión en la boca de los pacientes. El objetivo del presente trabajo fue \\ estudiar la corrosión de aparatos de ortodoncia fabricados con acero inoxidable con soldadura por \\ punto o por aporte de aleación de plata. Los resultados obtenidos mostraron que cuando el acero \\ inoxidable contiene aportes de soldadura de material de base plata, ésta actúa como ánodo \\ disolviéndose preferencialmente a una velocidad considerable. Los aparatos de ortodoncia \\ construidos con acero inoxidable soldado con aporte de material de base plata, corren el riesgo de \\ destrucción debido a un ataque intenso en el cordón de soldadura con el consiguiente despegue de las \\ piezas unidas.
}

Palabras clave: Aleaciones dentales. Acero inoxidạble. Aleación de plata. Corrosión.

\section{Corrosión of weldments in orthodontic appliances}

\begin{abstract}
The study of corrosion-related problems of dental materials has undergone a considerable development in recent years in order to avoid the use of materials with insufficient corrosion resistance in patients' mouth. The subject of the present work was to study a particular type of corrosion: galvanic corrosion. One of the most common case of galvanic couples in patients' mouth are the orthodontic appliances. The materials studied in the present work were stainless steel strips and silver-copper wires, isolated and welded between them. The electrochemical tests were performed in a $\mathrm{NaCl} 0.1 \mathrm{M}$ and Lactic Acid $0.1 \mathrm{M}$ solution ( $\mathrm{pH} 2.3$ ), and after test, the specimens were observed using the optical and scanning electron microscope. The results show that when stainless steel is coupled with a silver solder, the last is the anode of the galvanic couple. As a consequence of this, the silver solder undergone a severe attack. Stainless steel orthodontic appliances with silver solder are feasibly destroyed due to a severe attack on the filler metal disjoining the welded parts.
\end{abstract}

Keywords: Dental alloys. Stainless steel. Silver alloy. Corrosion.

\section{INTRODUCCIÓN}

La ortodoncia es el estudio del crecimiento y evolución del sistema de masticación y de la prevención y tratamiento de las anormalidades en su desarrollo (1). Estos tratamientos se llevan a cabo, casi siempre,

(•) Trabajo recibido el día 8 de enero de 1997.

(*) Laboratorio de Sólidos Amorfos. Facultad de Ingeniería. Universidad de Buenos Aires. Paseo Colón 850, (1063) Buenos Aires (Argentina).

(**) Fundación para el Estudio de los Materiales Dentales. Alvarado 121, (1704) Ramos Mejía. Buenos Aires (Argentina).

(***) CONICET y Comisión Nacional de Energía Atómica. Gerencia, Centro Atómico Constituyentes. Unidad de Actividad Materiales. Avda. del Libertador 8250, (1429) Buenos Aires (Argentina). mediante la utilización de aparatos que, al ejercer determinada fuerza, producen movimientos dentarios. Existen dos tipos de aparatos, los fijos y los que se pueden quitar y poner fácilmente. En el primer caso se fijan a los dientes y sólo pueden ser extraídos por un profesional, y en el segundo el paciente se los puede sacar y poner en la boca.

Dentro de los materiales empleados para la construcción de aparatos de ortodoncia, se encuentran distintas aleaciones que se utilizan en forma de alambres, bandas, tornillos y los denominados "brackets". Aunque se han empleado diferentes aleaciones con este propósito, el material utilizado más ampliamente es el acero inoxidable $18 \mathrm{Cr}-8 \mathrm{Ni}$, ya que cumple con adecuados requisitos mecánicos, químicos y biológicos.

En la construcción de los citados aparatos se emplean alambres y cintas que se comercializan al 
efecto, y que se han de soldar entre sí para dar forma definitiva a los aparatos (2). La mayoría de las operaciones de unión de las piezas de los aparatos de ortodoncia se lleva a cabo por medio de soldadura autógena, que consiste en la unión directa de dos materiales por medio de calor, presión, o por ambas, sin el aporte de ningún otro material. El acero inoxidable se puede soldar de esta forma utilizando presión mecánica a elevada temperatura con un procedimiento que se denomina soldadura por punto, técnica que consiste en hacer pasar una corriente eléctrica directamente a través de la partes a unir, generándose por la resistencia del acero suficiente calor para producir una fusión localizada. La presión aplicada para mantener a las partes en contacto favorece la unión. El calor generado por el material es proporcional al cuadrado de la intensidad de corriente aplicada, a la resistencia eléctrica de la aleación y al tiempo que dura el paso de corriente. Para mantener al acero inoxidable con adecuadas propiedades, el tiempo durante el cual se mantiene una temperatura elevada debe ser el mínimo posible a efectos de evitar (entre otras cosas), la precipitación de carburos de cromo en los límites de grano, que disminuyen la resistencia a la corrosión que presenta esta aleación en medios orales.

La unión de piezas de acero inoxidable también es posible mediante soldadura con material de aporte aleado, teniendo así la ventaja de no ser necesaria una temperatura elevada con lo que disminuyen los problemas producidos por el calentamiento de estos aceros. En este tipo de soldadura se emplea una aleación de bajo punto de fusión. Las aleaciones usadas más frecuentemente para soldar acero inoxidable están basadas en un contenido del 45-65 \% Ag, hasta un 25 $\% \mathrm{Cu}$ y cantidades variables de cinc. Es habitual que las temperaturas que se emplean en este tipo de soldadura no superen los $650{ }^{\circ} \mathrm{C}$, evitando con ello la peligrosa precipitación de carburos de cromo en los límites de grano ya que, aunque la temperatura es suficiente para provocar la precipitación, el tiempo de permanencia a dicha temperatura no lo es. Sin embargo, la existencia de una aleación $\mathrm{Ag}-\mathrm{Cu}-\mathrm{Zn}$ uniendo las piezas de acero inoxidable implica una disminución de la resistencia a la corrosión, debida, fundamentalmente, a la formación de pares galvánicos.

El objetivo del presente trabajo es estudiar comparativamente la resistencia a la corrosión que presentan, en medios orales, los aceros inoxidables empleados en la construcción de aparatos de ortodoncia sometidos a soldadura autógena y con aporte de aleación, con el fin de que se pueda predecir la vida útil de dichos aparatos.

\section{TÉCNICA EXPERIMENTAL}

Los materiales empleados en el presente trabajo fueron acero inoxidable tipo AISI 304 y aleación para soldadura de base plata. El acero inoxidable para aparatos de ortodoncia utilizado se presenta en forma de cintas de $8 \mathrm{~mm}$ de ancho y $0,2 \mathrm{~mm}$ de espesor, y el material de aporte en forma de alambres de 0,8 $\mathrm{mm}$ de diámetro. Las composiciones químicas de ambos materiales se muestran en la tabla I.

Las cintas de acero inoxidable se ensayaron de tres formas: en estado de recepción de fábrica, unidas entre sí por medio de soldadura autógena (por puntos), y soldadas con aporte de aleación de plata. En la figura 1 se muestra una micrografía de las probetas de acero inoxidable soldadas por puntos. El revelado de la microestructura (3) muestra que a ambos lados de la unión se observa la precipitación de carburos de cromo y la presencia de las llamadas "gotas frías", producto de una inadecuada selección de la tensión o de la intensidad de corriente durante la realización de la soldadura. La presencia de estos defectos será de fundamental importancia para interpretar la susceptibilidad a la corrosión que presentan estas soldaduras.

Las probetas obtenidas con las técnicas descritas se pulieron y se montaron adecuadamente para efectuar estudios electroquímicos (curvas de polarización) (4). Se estableció en cada una un contacto eléctrico a través de un alambre de cobre que se hizo pasar por un tubo de vidrio $\mathrm{y}$, posteriormente, se cubrieron con resina epoxi, dejando un área libre expuesta de aproximadamente $1 \mathrm{~cm}^{2}$. Las muestras

TABLA I.- Composición química de los materiales empleados en el presente trabajo, \% en masa

TABLE I.- Chemical composition of the alloys used in the present work, \% in mass

\begin{tabular}{|c|c|c|}
\hline Elemento & Acero inoxidable & $\begin{array}{c}\text { Aleación de } \\
\text { soldadura }\end{array}$ \\
\hline $\mathrm{Ni}$ & $11,5 \pm 0,6$ & $<0,005$ \\
$\mathrm{Cr}$ & $18,3 \pm 0,1$ & - \\
$\mathrm{Mo}$ & $0,28 \pm 0,02$ & - \\
$\mathrm{Si}$ & $\approx 0,05$ & $\approx 0,002$ \\
$\mathrm{Fe}$ & balance & $\approx 0,005$ \\
$\mathrm{Al}$ & $\approx 0,002$ & $\approx 0,002$ \\
$\mathrm{Cu}$ & $0,05-0,2$ & $13,5 \pm 1,7$ \\
$\mathrm{Mn}$ & $\approx 0,05$ & - \\
$\mathrm{Mg}$ & $<0,0002$ & $<0,0002$ \\
$\mathrm{~V}$ & $0,02-0,05$ & - \\
$\mathrm{C}$ & $0,051 \pm 0,0002$ & - \\
$\mathrm{S}$ & $0,0055 \pm 0,0002$ & - \\
$\mathrm{Zn}$ & - & $14,5 \pm 0,9$ \\
$\mathrm{Ag}$ & - & balance \\
\hline
\end{tabular}




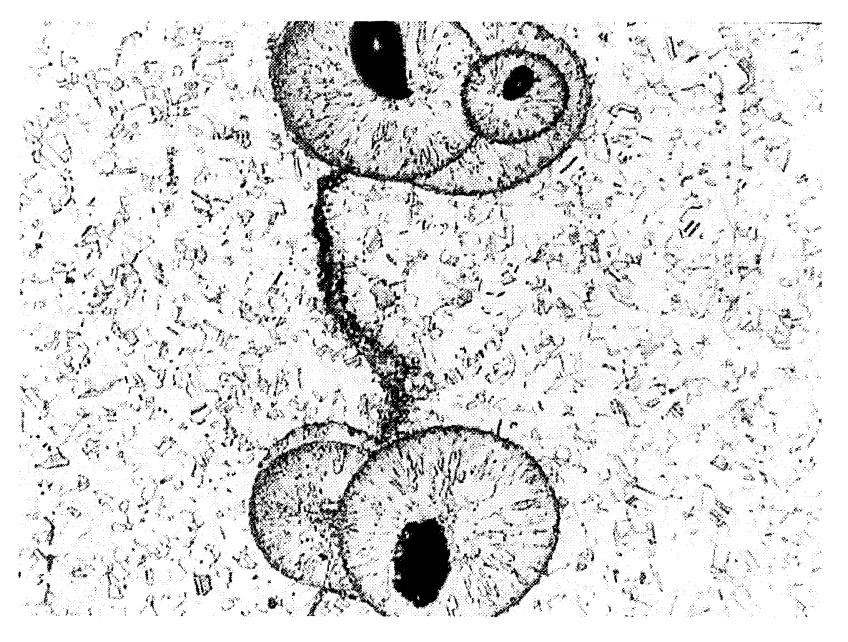

FIG. 1.- Micrografía del acero inoxidable tras la aplicación de soldadura por punto. Se observa la presencia de precipitados de carburos de cromo y de "gotas frías". $\times 500$.

FIG. 1.- Stainless steel micrograph after weld spot. Chromiun carbide precipitates and spatter are seeing. $\times 500$.

así montadas se desengrasaron con acetato de etilo y se secaron con aire caliente. Los ensayos electroquímicos se realizaron en una solución $0,1 \mathrm{M} \mathrm{NaCl}+$ $0,1 \mathrm{M}$ ácido láctico (5), con un valor de $\mathrm{pH}$ de 2,3. Esta solución se preparó con reactivos de grado analítico y agua de elevada pureza $(18 \mathrm{M} \Omega \cdot \mathrm{cm}$ de resistividad). Los potenciales de electrodo que se citan en este trabajo se determinaron empleando un electrodo de calomelanos saturado (ecs) con referencia a la escala del electrodo normal de hidrógeno (enh).

Los ensayos consistieron en el trazado de las curvas de polarización potenciocinéticas (por triplicado), comenzando con un potencial inferior al correspondiente de corrosión $\left(E_{\text {corr }}\right)$, a una velocidad de barrido de $0,5 \mathrm{mV} \cdot \mathrm{s}^{-1}$. Antes de introducir las probetas en la solución, ésta se desaireó durante 90 min mediante burbujeo de nitrógeno purificado. Previamente al inicio de las mediciones, se permitía que las muestras alcanzaran un potencial de corrosión cuasi estacionario por exposición a la solución a temperatura ambiente durante $1 \mathrm{~h}$ a circuito abierto con burbujeo de nitrógeno. Las medidas se llevaron a cabo a temperatura ambiente con un potenciostato PAR 176 y un equipo de barrido de potenciales PAR 175, obteniéndose el registro gráfico de las curvas mediante un equipo Houston X-Y Recorder. Para los ensayos se empleó una celda convencional de tres electrodos (6) con una capacidad de $50 \mathrm{~cm}^{3}$. Tras los ensayos, las probetas se observaron con un microscopio óptico y con otro electrónico de barrido (MEB) Philips SEM 500.

De forma complementaria, se trazaron las curvas de polarización de la aleación de soldadura y de los metales puros que la constituyen (plata, cobre y cinc) a efectos de determinar cuál es el efecto que cada uno de ellos tiene sobre la conducta de la aleación.

También se llevó a cabo el estudio del comportamiento del par galvánico acero inoxidable-aleación de soldadura, sumergiendo en la solución $\mathrm{NaCl}$ $0,1 \mathrm{M}$ + ácido láctico $0,1 \mathrm{M}$ aireada naturalmente, una probeta de acero inoxidable y otra de la aleación de soldadura que se unieron a través de un circuito externo en el cual se intercaló un microamperímetro Keithley para medir la corriente galvánica generada que se representó gráficamente en función del tiempo. En la misma solución se introdujo también una probeta de acero inoxidable en la que se había depositado parcialmente material aleado de soldadura, y un electrodo de referencia de calomelanos saturado con el cual se midió la evolución de los potenciales de todas las probetas en función del tiempo. Tras los ensayos, estas probetas se observaron con MEB.

\section{RESULTADOS Y DISCUSIÓN}

La curva de polarización del acero inoxidable en solución de $\mathrm{NaCl} 0,1 \mathrm{M}$ + ácido láctico $0,1 \mathrm{M}$ se muestra en la figura 2. El potencial de corrosión es de aproximadamente $0,050 \mathrm{~V}_{\mathrm{enh}}$. A partir de dicho potencial, la corriente asciende hasta llegar a una amplia zona pasiva (de casi $0,500 \mathrm{~V}$ de extensión) con intensidades menores que $1 \mu \mathrm{A} \cdot \mathrm{cm}^{-2}$. Al llegar a $0,525 \mathrm{~V}_{\text {enh }}$, la corriente sube abruptamente debido a la ruptura de la pasividad, dando origen al picado generalizado de la probeta. La observación de la misma con MEB muestra la presencia de gran cantidad de picaduras de morfología irregular.

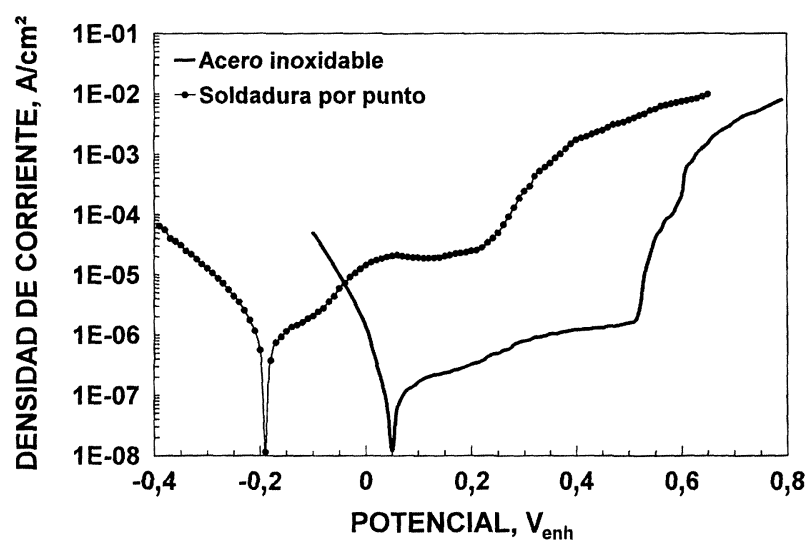

FIG. 2.- Curvas de polarización del acero inoxidable sin y con soldadura por punto en solución $\mathrm{NaCl}$ $0,1 \mathrm{M}+$ ácido láctico $0,1 \mathrm{M}$ a temperatura ambiente.

FIG. 2.- Polarization curves of stainless steel with and without weld spot, in $0.1 \mathrm{M} \mathrm{NaCl}+0.1 \mathrm{M}$ lactic acid solution at room temperature. 
La figura 2 incluye también la curva de polarización de la zona correspondiente a la soldadura autógena efectuada sobre el acero inoxidable (soldadura por punto). El potencial de corrosión (aprox. $-0,200 \mathrm{~V}_{\text {enh }}$ ) es menor (más catódico) con respecto al obtenido con el material sin soldar. A partir del mismo, la densidad de corriente asciende hasta alcanzar una zona en la que se transforma en independiente del potencial aplicado, con valores del orden de $10 \mu \mathrm{A} \cdot \mathrm{cm}^{-2}$, hasta que al llegar a 0,250 $\mathrm{V}_{\text {enh }}$ la densidad de corriente asciende gradualmente. La observación con MEB del material ensayado (Figs. 3 y 4) muestra que en la zona correspondiente a la unión de las dos cintas se produce corrosión

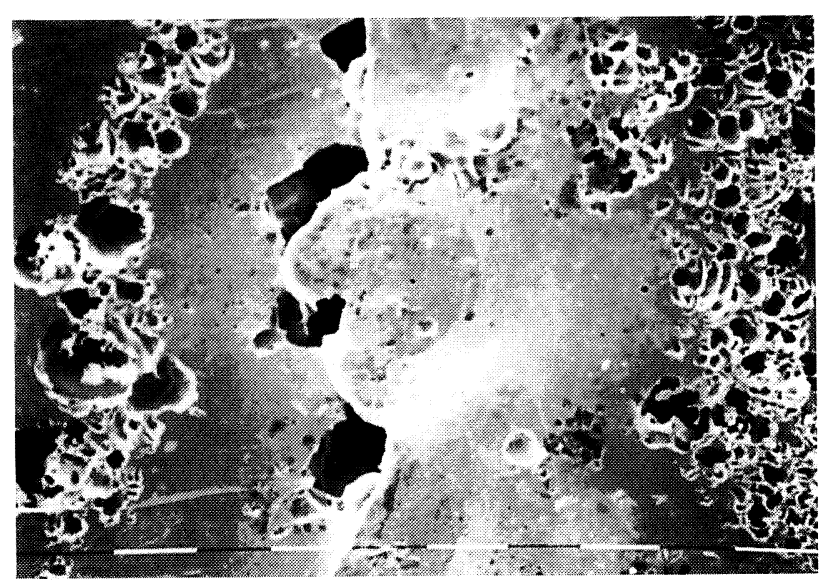

FIG. 3.- Micrografía de la zona correspondiente a la soldadura por punto sobre el acero inoxidable, tras el trazado de la curva de polarización (líneas: $10 \mu \mathrm{m}$ ).

FIG. 3.- Micrograph of stainless steel in weld spot zone after polarization curve (dashes: $10 \mu \mathrm{m}$ ).

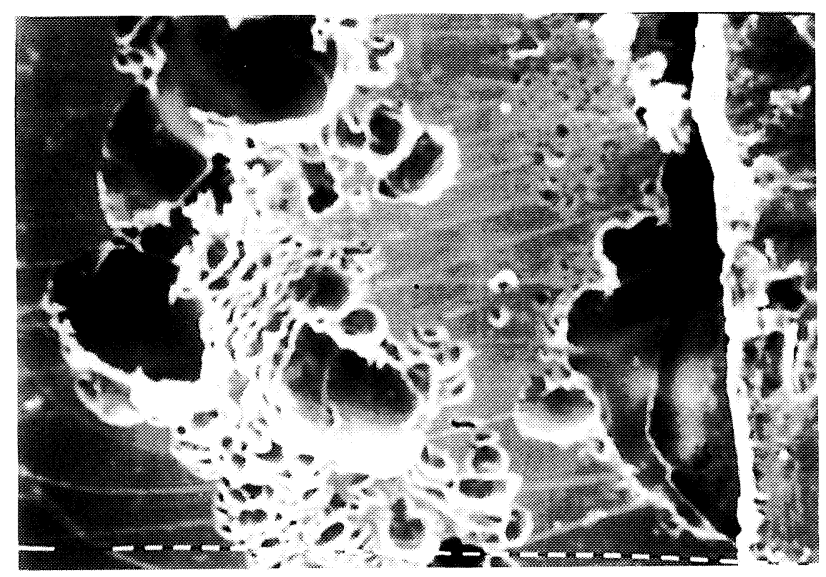

FIG. 4.- Detalle de la zona correspondiente a la soldadura por punto donde se observa el ataque producido durante el trazado de la curva de polarización (líneas: $10 \mu \mathrm{m}$ ).

FIG. 4.- Corrosion attack on stainless steel weld spot zone, after polarization curve (dashes: $10 \mu \mathrm{m})$. por resquicios, mientras que en una zona a ambos lados de la misma (de aproximadamente $5 \mathrm{~mm}$ ) el material presenta con profusión picaduras irregulares. El ataque corrosivo comienza en las zonas donde se encuentran con mayor densidad y continuidad los precipitados de carburos de cromo y en las zonas donde se depositan las "gotas frías".

En la figura 5 se muestran las curvas de polarización del material de soldadura y de sus componentes aislados, obtenidas en solución de $\mathrm{NaCl}$ $0,1 \mathrm{M}+$ ácido láctico $0,1 \mathrm{M}$. El cinc puro presenta un potencial de corrosión de $-0,750 \mathrm{~V}_{\text {enh }}$, a partir del cual la densidad de corriente sube abruptamente hasta que, al superar un sobrepotencial de $250 \mathrm{mV}$, la densidad de corriente se transforma en independiente del potencial con un valor del orden de 30 $\mathrm{mA} \cdot \mathrm{cm}^{-2}$. El cobre presenta un potencial de corrosión de aproximadamente $0 \mathrm{~V}_{\text {enh }}$, a partir del cual se produce un monótono ascenso de la densidad de corriente. Por su parte, la plata tiene un potencial de corrosión de $0,150 \mathrm{~V}_{\text {enh }}$; en este caso, la densidad de corriente crece, presenta un máximo local y vuelve a subir a partir de $0,3 \mathrm{~V}_{\text {enh }}$ (valor coincidente con el potencial de formación de $\mathrm{AgCl}$ en soluciones $0,1 \mathrm{M}$ de cloruros) hasta que el valor de la densidad de corriente se hace independiente del potencial aplicado, con un valor de aproximadamente $5 \mathrm{~mA} \cdot \mathrm{cm}^{-2}$. En esta zona se produce el crecimiento de la película de $\mathrm{AgCl}$, y la independencia de la densidad de corriente con el potencial se debe a que este crecimiento está controlado por la difusión en estado sólido de la plata a través de la película de $\mathrm{AgCl}$ (7). Finalmente, la aleación de soldadura presenta un potencial de corrosión de $-0,220$ $\mathrm{V}_{\text {enh }}$; aquí la densidad de corriente sube hasta

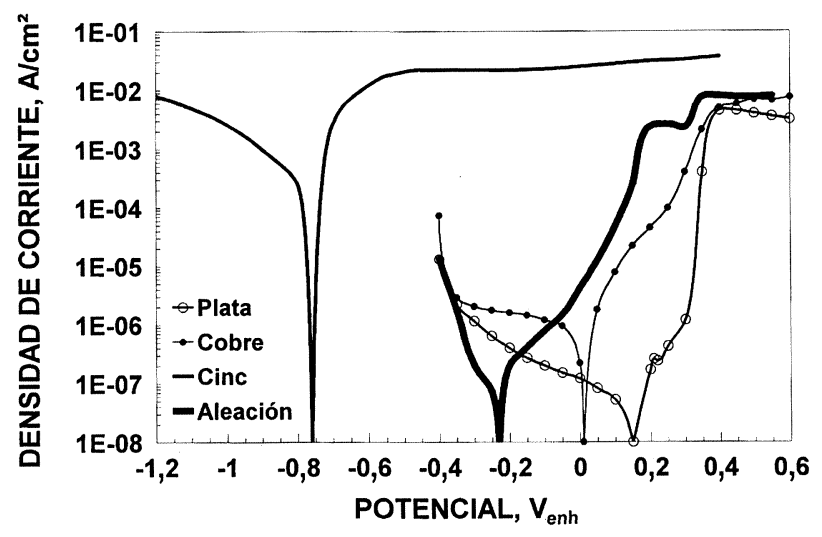

FIG. 5.- Curvas de polarización de la aleación de soldadura y de sus componentes aislados, en solución $\mathrm{NaCl} 0,1 \mathrm{M}$ + ácido láctico $0,1 \mathrm{M}$ a temperatura ambiente.

FIG. 5.- Polarization curves of weld filler alloy and its elemental components in $0.1 \mathrm{M} \mathrm{NaCl}+0.1 \mathrm{M}$ lactic acid at room temperature. 
llegar a $0,2 \mathrm{~V}_{\text {enh }}$, donde se estabiliza en $3 \mathrm{~mA}$. $\mathrm{cm}^{-2}$ durante $100 \mathrm{mV}$ del barrido de potenciales hasta que el llegar a $0,3 \mathrm{~V}_{\text {enh }}$ vuelve a subir, esta vez un orden de magnitud, para a partir de allí transformarse en independiente del potencial aplicado. De la comparación de las cuatro curvas de polarización presentadas, se deduce que el potencial de corrosión de la aleación está acusadamente influenciado por la presencia del cinc en su composición, que hace desplazarse dicho potencial a valores más catódicos con respecto a los que presentan el cobre y la plata pura en dicha solución. El comportamiento de la aleación a elevados potenciales refleja lo que ocurre con la plata pura, ya que el ascenso final obtenido a $0,3 \mathrm{~V}_{\text {enh }}$ coincide con el obtenido con dicho metal, mientras que la densidad de corriente constante a partir de 0,350 $\mathrm{V}_{\text {enh }}$ aproximadamente, se debe al engrosamiento de la película de $\mathrm{AgCl}$.

En la figura 6 se pueden apreciar las curvas de polarización del acero inoxidable, de la aleación de soldadura y de una probeta de acero inoxidable en la que se ha llevado a cabo un aporte de la aleación de soldadura (par galvánico), con una relación de áreas de 5:1 (aproximada a las condiciones reales). De dicha figura se deduce que el comportamiento del par galvánico se asemeja al de la aleación de soldadura, con un potencial de corrosión de $-0,175$ $\mathrm{V}_{\text {enh }}$ y un monótono ascenso de la densidad de corriente con el potencial aplicado hasta aproximadamente $0,2 \mathrm{~V}_{\text {enh }}$, donde se observa una zona de densidad de corriente constante, seguida de otra con

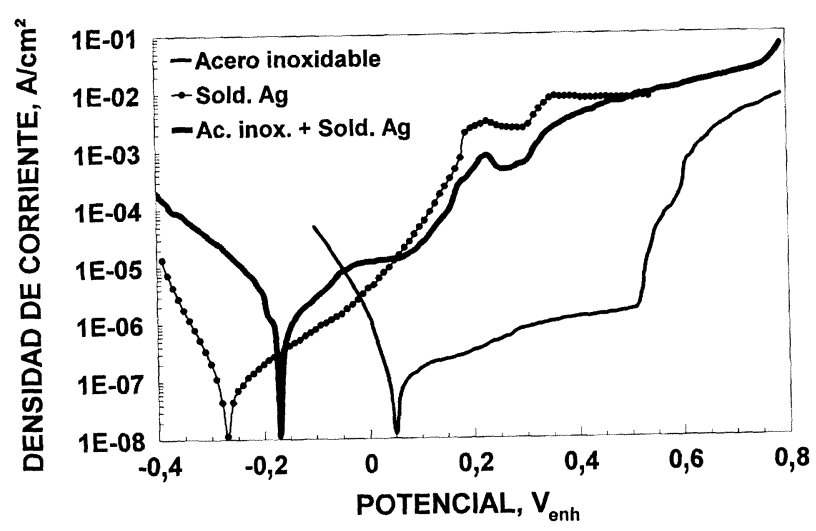

FIG. 6.- Curvas de polarización del acero inoxidable, de la aleación de soldadura y del acero inoxidable con aporte de aleación de soldadura, en solución $\mathrm{NaCl} 0,1 \mathrm{M}$ + ácido láctico $0,1 \mathrm{M}$ a temperatura ambiente.

FIG. 6.-Polarization curves of stainless steel, weld filler alloy and a welded stainless steel with filler alloy, in $0.1 \mathrm{M} \mathrm{NaCl}+0.1 \mathrm{M}$ lactic acid at room temperature. incremento de corriente, para finalizar con una zona en la cual la densidad de corriente es independiente del potencial aplicado. La observación con MEB de las probetas empleadas muestra la presencia de ataque preferencial sobre al material de aporte, que parece seguir planos cristalinos definidos.

En la figura 7 se observa el comportamiento del par galvánico acero inoxidable-aleación de soldadura con la relación de áreas 5:1 en función del tiempo. Durante las tres primeras horas de ensayo se obtuvieron las representaciones gráficas de la evolución del potencial de corrosión de la probeta de acero inoxidable y de la aleación de soldadura. En ambos casos se observó una disminución de este potencial con el tiempo. Para el caso del acero inoxidable se parte de $0,530 \mathrm{~V}_{\text {enh }}$ que, después de $3 \mathrm{~h}$, llega a $0,430 \mathrm{~V}_{\text {enh. }}$. Para la aleación de soldadura se parte de $0,160 \mathrm{~V}_{\text {enh }} \mathrm{y}$, transcurrido el mismo tiempo, el potencial de corrosión es de $0,050 \mathrm{~V}_{\text {enh }}$. Después del período de $3 \mathrm{~h}$, se cortocircuitaron las probetas intercalando un miliamperímetro de baja impedancia y, simultáneamente, se midió la evolución del potencial de corrosión del par galvánico así generado. Este último parte de un potencial de corrosión similar al alcanzado por la probeta de aleación de soldadura $\left(0,050 \mathrm{~V}_{\text {enh }}\right)$ y aumenta con el tiempo hasta llegar a $0,120 \mathrm{~V}_{\text {enh }}$ tras de 4,5 días de ensayo. De forma paralela, se siguió la evolución del potencial de corrosión de un par galvánico generado por el aporte de aleación de soldadura sobre una probeta de acero inoxidable, observándose que a medida que transcurre el ensayo los potenciales de corrosión de ambos pares galvánicos

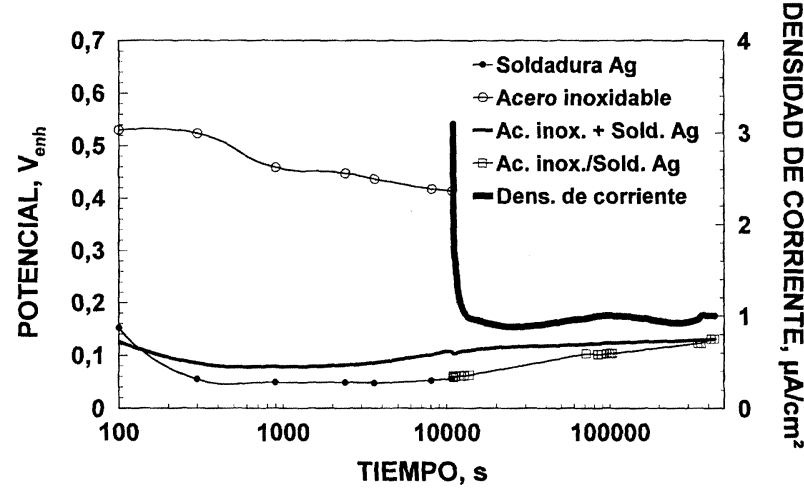

Fig. 7.- Evolución de los potenciales de corrosión y de la intensidad de corriente generada por el par galvánico acero inoxidable-aleación de soldadura para una relación de áreas 5:1, en solución $\mathrm{NaCl}$ $0,1 \mathrm{M}+$ ácido láctico $0,1 \mathrm{M}$ aireada.

FIG. 7.-Corrosion potentials and galvanic currents evolution in a stainless steel-filler metal alloy galvanic pair with a 5:1 area ratio, in 0.1M $\mathrm{NaCl}+0.1 \mathrm{M}$ lactic acid aereated solution at room temperature. 
tienden a igualarse. A su vez, la intensidad de corriente generada por el par galvánico surgido del cortocircuito de las probetas de ambos materiales parte de $3 \mu \mathrm{A} \cdot \mathrm{cm}^{-2}$ y desciende abruptamente tras las primeras horas de ensayo hasta estabilizarse en aproximadamente $1 \mu \mathrm{A} \cdot \mathrm{cm}^{-2}$ durante el resto del ensayo, siendo la aleación de soldadura el material que actúa como ánodo. La observación con MEB de los materiales ensayados mostró que, en ambos pares galvánicos, la zona correspondiente a la aleación de plata se encuentra muy atacada por una corrosión que parece seguir planos cristalinos preferenciales (Fig. 8). Al convertir los valores de densidad de corriente galvánica presentados en la figura 7 en velocidad lineal de ataque mediante la ley de Faraday (8 y 9), se encuentra que la velocidad de corrosión en estado estacionario de la aleación de soldadura es de $0,02 \mathrm{~mm} /$ año, valor que para otros sistemas puede resultar inofensivo, pero en este caso, tratándose de soldaduras cuyo espesor difícilmente supera algunas décimas de milímetro, se trata de una corrosión que consume más del $20 \%$ del material por año de uso, lo que se agrava por el

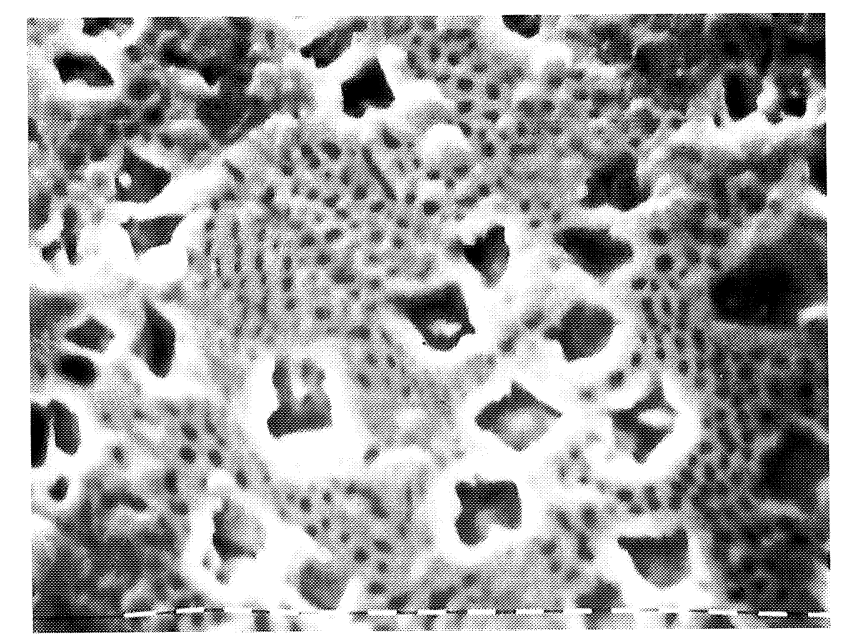

FIG. 8.- Micrografía de la zona correspondiente a la aleación de soldadura, después de someterla al par galvánico con el acero inoxidable durante 4,5 días. Se aprecia la presencia de ataque que sigue planos cristalinos definidos (líneas: $10 \mu \mathrm{m}$ ).

FIG. 8.- Micrograph of filler metal alloy after 4.5 days galvanic test. Crystalographic etching is detected (dashes: $10 \mu \mathrm{m}$ ). hecho de que en muchos pares galvánicos la corriente de corrosión puede ser significativamente mayor que la debida al macropar galvánico y a que los aparatos de ortodoncia se encuentran sometidos continuamente a tensiones mecánicas. Esta combinación de circunstancias lleva a la rotura temprana de los mismos.

\section{CONCLUSIONES}

A partir de los resultados obtenidos en el presente trabajo, se concluye que los aparatos de ortodoncia construidos con acero inoxidable utilizando soldadura por punto, se pueden despegar en caso de encontrarse precipitados continuos de carburos de cromo en los límites de grano, o la presencia de "gotas frías" debida a la mala selección de las condiciones de soldadura. Por otra parte, si se emplea soldeo con aporte de aleación de plata, el riesgo de despegue se debe a la corrosión selectiva que sufre el cordón de soldadura, agravado por el hecho de que la relación de áreas entre ánodo y cátodo en este caso es mucho menor que uno, siendo esta relación la más desfavorable para el caso de pares galvánicos (9).

\section{REFERENCIAS}

(1) Williams, D.F. y Cunningham, J. Materiales en la odontología clínica. Ed. Mundi, $1^{\text {a }}$ Ed. Buenos Aires, 1982: 335-351.

(2) Mueller, H.J. Tarnish and Corrosion of Dental Alloy. ASM Handbook. Vol. 13 (Corrosion). ASM International (9th Ed.), 1992: 1.354.

(3) Norma ASTM-A $26255 \mathrm{~T}$.

(4) Riesgo, O., BiAnchi, G.L. y Duffo, G.F. Rev. Metal. CENIM, 28 (4), 1992: 215-218.

(5) Geiss-Gerstorfer, J. J. Dent., 22, 1994: 247-251.

(6) de De Micheli, S.M. y Riesgo, O. Rev. Odonto-Stomatol., 7, 1978: 349-353.

(7) Duffo, G.S. Movilidad superficial en la corrosión bajo tensión. Tesis Doctoral. Facultad de Ciencias Exactas y Naturales. Universidad de Buenos Aires, 1990: 86-93.

(8) Fontana, M.G. Corrosion Engineering. 3rd Ed. McGrawHill, Inc. Nueva York, 1986: 173.

(9) Galvele, J.R. y Duffo, G.S. Procesos de Corrosión. Instituto de Tecnología, CNEA-UNSaM, IT-51/95. Buenos Aires, 1995: 4.17-4.18. 